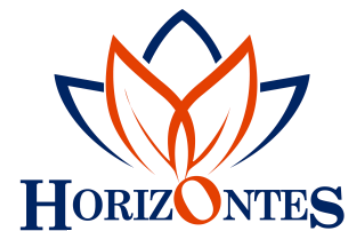

Horizontes. Revista de Investigación en Ciencias de la Educación Https://doi.org/10.33996/revistahorizontes.v5i18.152

Abril - junio 2021

Volumen 5 / No. 18

ISSN: $2616-7964$

ISSN-L: 2616 - 7964 pp. $354-372$

www.revistahorizontes.org

\title{
Abordaje epistemológico en la investigación educativa para la aproximación, constructo, modelo o perspectiva teórica
}

\author{
Epistemological approach in educational research for the approach, construction, model \\ or theoretical perspective
}
Abordagem epistemológica na pesquisa educacional pela abordagem teórica, construção, modelo ou perspectiva

\section{ARTICULO GENERAL}

\author{
Luis Doubront \\ dr.doubront@gmail.com \\ https://orcid.org/0000-0003-4174-2169
}

Universidad Nacional Experimental Simón Rodríguez, Venezuela

Recibido 08 de enero 2021 | Arbitrado y aceptado 15 de febrero 2021 | Publicado en 01 abril 2021

\section{RESUMEN}

\begin{abstract}
La producción del conocimiento científico para los investigadores en formación, se ha convertido en una dificultad sobre los elementos que deben regir y orientar las investigaciones puras, pretendiendo proponer la validación de teorías, a la luz de recetas instrumentalistas inherentes a los enfoques metodológicos cuantitativos o cualitativos, sin tomar en cuenta la fundamentación del abordaje epistemológico como eje transversal del proceso investigativo. En este escenario, surge la investigación con el propósito de dilucidar el abordaje epistemológico de la investigación educativa para la aproximación, constructo, modelo o perspectiva teórica. La metodología empleada se enmarcó en un enfoque cualitativo, utilizando el método hermenéutico-critico que permitió interpretar la información en unidades de análisis a través de libros y artículos indexados. En este sentido, los hallazgos de la investigación reseñaron la importancia para demarcar los ejes procedimentales y los ejes orientadores de la investigación en una interrelación necesaria para la producción del conocimiento científico. En este contexto, se concluye que la legitimidad de la investigación educativa obedece a la coherencia interna que se expresa en el abordaje epistemológico, ontológico y metodológico de la investigación.
\end{abstract}

Palabras clave: Abordaje; EpistemológicoInvestigación Educativa-Coherencia; Interna
ABSTRACT

The production of scientific knowledge for researchers in training has become a difficulty regarding the elements that should govern and guide pure research, seeking to propose the validation of theories, in the light of instrumentalist recipes inherent to quantitative or qualitative methodological approaches. , without taking into account the foundation of the epistemological approach as a transversal axis of the investigative process. In this scenario, research arises with the purpose of elucidating the epistemological approach of educational research for the theoretical approach, construct, model or perspective. The methodology used was framed in a qualitative approach, using the hermeneutical-critical method that allowed interpreting the information in units of analysis through indexed books and articles. In this sense, the research findings highlighted the importance of demarcating the procedural axes and the guiding axes of the research in a necessary interrelation for the production of scientific knowledge. In this context, it is concluded that the legitimacy of educational research obeys the internal coherence that is expressed in the epistemological, ontological and methodological approach of the research.

Key words: Epistemological; ApproachEducational; Research-Internal; Coherence

\section{RESUMO}

A produção de conhecimento científico para pesquisadores em formação tem se tornado uma dificuldade quanto aos elementos que devem reger e nortear a pesquisa pura, buscando propor a validação de teorias, à luz de receitas instrumentalistas inerentes às abordagens metodológicas quantitativas ou qualitativas. Sem levar em consideração conta a fundamentação da abordagem epistemológica como eixo transversal do processo investigativo. Nesse cenário, a pesquisa surge com o propósito de elucidar a abordagem epistemológica da pesquisa educacional para a abordagem teórica, construto, modelo ou perspectiva. A metodologia utilizada enquadrou-se numa abordagem qualitativa, utilizando o método hermenêutico-crítico que permitiu interpretar as informações em unidades de análise através de livros e artigos indexados. Nesse sentido, os achados da pesquisa destacaram a importância de demarcar os eixos procedimentais e os eixos norteadores da pesquisa em uma inter-relação necessária à produção do conhecimento científico. Nesse contexto, conclui-se que a legitimidade da pesquisa educacional obedece à coerência interna que se expressa na abordagem epistemológica, ontológica e metodológica da pesquisa.

Palavras-chave: Abordagem; PesquisaCoerência Epistemológica-Educacional; interno 


\section{INTRODUCCIÓN}

Hablar de abordaje epistemológico en la investigación, se convierte en una necesidad en la naturaleza de las ciencias sociales, ya que muchos autores aseguran que la producción del conocimiento obedece a una teoría del conocimiento científico que rige la investigación, según (Paz-Sandín, 2003; p. 47). "Una perspectiva epistemológica es una forma de comprender y explicar cómo conocemos lo que sabemos: ¿Qué tipo de conocimiento obtendremos en una investigación? ¿Qué características tendrá ese conocimiento? ¿Qué valor puede otorgarse a los resultados obtenidos?". Por su parte, Crotty (1998, p.7) identifica tres orientaciones epistemológicas fundamentales circunscritas en los paradigmas o perspectivas teóricas de la investigación: "el objetivismo, el construccionismo y el subjetivismo".

En este escenario, la formación de investigadores evidencia un cúmulo de dificultades conceptuales para la producción del conocimiento científico, siendo recurrente escuchar en los pasillos de las universidades hablar más de investigación cuantitativa y cualitativa desatendiendo el abordaje epistemológico como eje orientador del proceso investigativo. Según, (Hughes, 1980: p.13; Citado por Corbetta 2007). "Cada procedimiento o instrumento de investigación está entrelazado de manera inextricable con las interpretaciones concretas del mundo que tiene el investigador y su modo de conocer ese mundo". Así pues, esta consideración en ocasiones es omitida por diferentes autores, expresando debates entre la "epistemología vs metodología" entre lo "cuantitativo y lo cualitativo".

La discusión de fondo entre métodos cuantitativos y cualitativos no es metodológica. Sólo tiene sentido planteada epistemológicamente y sólo desde la epistemología puede ser resuelta. Y, desde ésta, se advierte que la cuestión no está ni en lo cualitativo ni en lo cuantitativo sino en cómo se relacionan ambas vertientes de lo real. Justamente esta relación se encuentra hoy en un proceso de cambio, (Munné, 1995: VI; Citado por Paz Sandín 2003).

Las divisiones epistemológicas y de paradigmas no contribuyen en nada a la ciencia, (salvo que se utilicen con fines clasificatorios o taxonómicos), ya que la complejidad de los problemas que aquejan a la sociedad humana son muchos y cada vez más de consecuencias inciertas, (Ortiz, 2013; p.18). En atención a lo expuesto, se reflejan contradicciones sobre el eje orientador del proceso investigativo, por un lado, se expresa que el enfoque metodológico obedece a la posición del investigador a través de la epistemología y por el otro se les resta valor a las diversas fuentes epistemológicas privilegiando la metodología de la investigación.

En el modelo que estamos siguiendo aquí, notará que la distinción entre la investigación cualitativa y la investigación cuantitativa ocurre en el nivel de métodos. No ocurre a nivel epistemológico o teórico perspectiva. Lo que ocurre allá atrás en esos niveles elevados es una distinción entre la investigación objetivista/positivista, por un lado, e investigación construccionista o subjetivista, por el otro. Sin embargo, en la mayoría de libros de texto en investigación, es investigación cualitativa e investigación cuantitativa que se oponen entre sí como polos opuestos, (Crotty 1998; p.15).

En este escenario, la investigación educativa se ha reducido a un debate que conlleva a una fragmentación del conocimiento, donde el abordaje epistemológico se considera como un ente aislado del proceso donde la 
relación del sujeto-investigador para conocer la realidad, se ha reducido históricamente a límites del pensamiento "cuantitativos o cualitativos", generando una interpretación metodológica-técnica para el abordaje de la investigación que carece de fundamentos conceptuales para justificar la legitimidad en la producción científica, en consecuencia las formaciones discursivas para una aproximación, modelo, constructo o perspectiva teórica; quedan relegadas a un modismo de estilo por parte de los investigadores.

Michael Foucault (1979) en su obra la "arqueología del saber", establece que un investigador debe ofrecer un análisis lógico en el modo de proceder. "Analizar positividades, es mostrar de acuerdo con qué reglas una práctica discursiva puede formar grupos de objetos, conjuntos de enunciaciones, juegos de conceptos, series de elecciones teóricas", (p.305).

Foucault marca sus herramientas con su signo especial: reflexión sobre la producción de conocimientos científicos, en ese sentido podemos hablar de una epistemología que, en tanto proceso para la producción de conocimientos no le rinde cuentas al cientificismo. La epistemología es vía obligada para hacer teoría, (Perdomo, 2009; p. 136).

En atención a lo expuesto, la problemática se reduce en la necesidad de cuestionar y clarificar elementos teóricos conceptuales para abordar la investigación educativa, tomando en cuenta la necesidad de incorporar los símbolos significantes del lenguaje en la línea discursiva del investigador para la acción comunicativa. Es por ello, la necesidad de dilucidar ¿Cuál es el abordaje epistemológico de la investigación educativa para la aproximación, constructo, modelo o perspectiva teórica?, permitiendo interpretar la percepción de la realidad social por parte del investigador, la relación entre el investigador y lo investigado, al igual; que la estrategia procedimental para legitimar los axiomas de producción científica.

\section{METODOLOGÍA}

El artículo de investigación se desarrolló a través de un abordaje epistemológico racionalidealista como eje orientador del proceso, utilizando como método el hermenéutico crítico como eje procedimental. Al enfoque racional-idealista, según el cual no necesariamente debería cubrirse trabajo de campo, permitiendo como fundamento de este enfoque, las expresiones vivencial interpretativas, reflexivistas, las expresiones de la teoría crítica, la neo-dialéctica, así como todo lo que se oriente bajo la concepción del constructivismo, es decir, el reforzamiento de constructos teóricos o conocimientos que ya se poseen, (De Berrios \& Briceño, 2009; p.49).

Para los hermenéuticos críticos, la representación del significado de una expresión es relativamente no problemática. Lo importante es la valoración de los significados de dicha expresión a la luz de condiciones históricas. La investigación se ocupa de clarificar las condiciones bajo las que una comprensión distorsionada puede haber ocurrido, una clarificación que debe conducir a una acción práctica emancipadora y de aquellas personas cuyas comprensiones han sido distorsionadas. Podemos citar los trabajos de J. Habermas como representantes de esta postura, (Paz-Sandín, 2003; p. 61).

En atención a lo expuesto, el artículo de investigación se desarrolló en un diseño documental utilizando fuentes (primarias y secundarias), tomando en cuenta obras reconocidas y artículos de investigación indexados en (Scielo, Scopus, Dialnet, Latindex, Redalyc), que permitió la recolección de la 
información a través de las unidades de análisis.

Para la investigación cualitativa, la investigación documental no sólo es una técnica de recolección y validación de información, sino que constituye una de sus estrategias, la cual cuenta con particularidades propias en el diseño del proyecto, la obtención de la información, el análisis y la interpretación; y como estrategia cualitativa, también combina diversas fuentes (primarias y secundarias), (Galeano, 2012; p.104).

En este sentido, se recolectó la información aplicando la técnica de revisión sistemática. La revisión sistemática se puede reconocer como un estudio, en sí mismo, en el cual el o los autores tienen una interrogante bien definida y estructurada; se detecta, obtiene, consulta y recopila información de datos relevantes sobre artículos previos y necesarios para contestar la pregunta de investigación. Se realiza un análisis y se genera una conclusión bajo un método ordenado y reproducible (Torres \& López, 2014, p. 394).

\section{HALLAZGOS DE LA INVESTIGACIÓN}

En este escenario los hallazgos de la investigación, se orientaron a través de un proceso de categorización, estructuración, contrastación y hermeneusis (teorización), en correspondencia con el diseño de la investigación y el enfoque cualitativo. En este sentido, para dilucidar el abordaje epistemológico en la investigación educativa se hizo necesario desmenuzar un conjunto de unidades de análisis que orientaron el proceso investigativo, reconociendo la expresión de diversos autores inherentes a la temática. El proceso de categorización se desarrolló a nivel hermenéutico subrayando las categorías emergentes, por su parte la estructuración se produjo al sistematizar la información recolectada a través de libros y artículos indexados; tomando en cuenta la posición investigativa, teórica y filosófica.

En concordancia, se contrastaron los resultados de la investigación aplicando una técnica de triangulación teórica para posteriormente generar una nueva perspectiva a través de la hermeneusis crítica. De igual manera, se desprendieron las unidades de análisis tomando en cuenta la pregunta central: ¿Cuál es el abordaje epistemológico de la investigación educativa para la aproximación, constructo, modelo o perspectiva teórica?

Al respecto, Guba y Lincoln (1998; p. 5) exponen que "Los paradigmas de investigación definen para los investigadores qué es lo que están haciendo y qué cae dentro y fuera de los límites de una investigación legítima". Por otra parte, para (Dendaluce, 1999; p. 365; Citado por Paz Sandín 2003), expone que "la dimensión epistemológica es una dimensión que muchos investigadores ni tan siquiera se plantean o que desatienden, cuando en realidad está en la base de muchos comportamientos y decisiones metodológicotécnicas posteriores" y Crotty (1998; p.3) define que "la Epistemología o teoría del conocimiento se encuentra incrustada en la teoría perspectiva (paradigma) y por tanto, en la metodología".

En atención a lo expuesto, el abordaje epistemológico en la investigación educativa no puede interpretarse como un ente aislado, ya que adoptar una posición epistemológica registra consecuencias en el abordaje investigativo que impacta en la percepción del investigador frente a la realidad social, la relación entre el investigador y lo investigado y la estrategia metodológica del investigador. En tal sentido se presentan las unidades de análisis (Cuadros 1, 2, 3, 4). 
Cuadro 1. Unidades de Análisis.

\begin{tabular}{ll}
\hline PERCEPCIÓN DEL INVESTIGADOR & PLANO ONTOLÓGICO \\
RELACIÓN DEL INVESTIGADOR & PLANO EPISTEMOLÓGICO \\
METODOLOGÍA DEL INVESTIGADOR & PLANO METODOLÓGICO \\
\hline
\end{tabular}

Fuente: Unidades de Análisis elaborada por el autor (2021). 
Cuadro 2. ¿Cuál es la percepción del investigador frente a la realidad social?

\section{POSICIÓN INVESTIGATIVA}

POSICIÓN TEÓRICA

\section{POSICIÓN FILOSÓFICA}

Fuente 1: Las convicciones acerca de las relaciones del sujeto-investigador con la realidad objeto observable se concretan igualmente en dos valores, como son, el "idealismo" y el "realismo", (De Berrios \& Briceño; 2008).

Fuente 2: Responder las preguntas ¿qué es la realidad? y ¿qué es la representación?, es un asunto eminentemente ontológico. En ese sentido, existen al menos dos corrientes de interpretación de la realidad: una de tipo positivista, natural, materialista $\mathrm{u}$ objetivista, y otra de tipo subjetiva, social, constructivista o intersubjetivista, (Hincapié; 2017).

Fuente 3: La primera hipótesis de carácter ontológico hace referencia directa a la interpretación de la realidad o de la verdad en la sociología en sus aspectos filosóficos, de la siguiente forma: la aparición de la corriente sociológica del interaccionismo simbólico a finales de la década de los años 60 , ha propiciado un cambio metodológico en las ciencias sociales y humanas sobre cómo se entiende la realidad y la verdad, de tal forma que la comprensión inicial de una realidad objetiva, se ha desplazado hacia una comprensión de una realidad interpretada y construida subjetivamente, (Vidal; 2013).
Fuente 6: La posición del positivismo de realismo ingenuo, al suponer una realidad externa objetiva sobre la cual pueden converger las investigaciones, al realismo crítico de postpositivismo, que aún supone una realidad objetiva pero que acepta que sólo se le puede comprender imperfecta y probabilísticamente, a realismo histórico de la teoría crítica, que supone una realidad comprensible que consiste en estructuras históricamente situadas que son, en la ausencia del "insight", tan limitantes como si fueran reales, al relativismo del constructivismo, que supone realidades sociales múltiples, comprensibles $y$, en ocasiones, opuestas, que son producto del intelecto humano, pero que pueden cambiar al volverse sus constructores más informados y sofisticados, (Guba y Lincoln; 1998).

Fuente 7: Realismo (una ontológica noción que afirma que las realidades existen fuera de la mente) a menudo se toma implicada al objetivismo (una noción epistemológica que afirma que el significado existe en los objetos independientemente de cualquier conciencia). En algunos casos Incluso encontramos el realismo identificado con el objetivismo.
Fuente 8: Al determinar al sujeto, el objeto se muestra independiente de él, trascendente a él. Todo conocimiento menta ("intende") un objeto, que es independiente de la conciencia cognoscente. El carácter de trascendentes es propio, por ende, a todos los objetos del conocimiento. Dividimos los objetos en reales e ideales. Llamamos real a todo lo que nos es dado en la experiencia externa o interna o se infiere de ella. Los objetos ideales se presentan, por el contrario, como irreales, como meramente pensados, (Hessen; 1999).

Fuente 9: Todas las concepciones filosóficas de la antigüedad grecorromana, tanto Platón como Aristóteles y del medievo cristiano son realistas, dan por sentado que existe una realidad fuera de la mente. Aristóteles llama a la realidad explicada anteriormente substancia, mientras que Platón la denomina idea. Por otra parte, Descartes y Hume son realistas en este sentido, puesto que creen que la ciencia no hace sino reflejar lo real exterior a la mente. (Severini; 1.961).

Fuente 10: Todas las posturas idealistas son modernas. Diferenciemos algunas corrientes idealistas: (a). Berkeley sostenía que no había realidad alguna fuera de la mente, pero no de nuestra mente, sino de la mente de dios. 


\section{POSICIÓN INVESTIGATIVA} POSICIÓN TEÓRICA

\section{POSICIÓN FILOSÓFICA}

Fuente 4: El primer punto de vista constituye, Guba y Lincoln (1994, pág. 108) ciertamente esencialmente, una interpretación Idealista del conocimiento científico, asociada a una base de subjetividad; mientras que el segundo, una interpretación Realista del conocimiento científico, asociado a una base de objetividad. Evidentemente, entre ambas posiciones hay matices intermedios que van desde el idealismo "absoluto o dogmático", hasta el "realismo ingenuo" y el "realismo crítico", (Camacho, Fortaines \& Urdaneta; 2005). postulan un vínculo necesario entre los dos

cuando afirman que si, por ejemplo, se asume una realidad "real", la postura del conocedor debe ser uno de desapego objetivo o libertad de valores para ser capaz de descubrir "cómo son realmente las cosas" y "cómo funcionan realmente las cosas". (Crotty 1998) (Chaves \& Gadea; 2018).

(b). Kant sostenía que lo real que llamamos el objeto era el producto de una síntesis entre las formas puras del sujeto y el contenido dado en la experiencia. El idealismo sostiene que el sujeto determina al objeto en la relación de conocimiento; solo podemos conocer aquello que se amolda a nuestras formas subjetivas de conocer. Esta postura se llama idealista, porque sostiene que es el pensamiento o idea lo que constituye a las cosas como objetos de conocimiento,

Nueva Perspectiva: Los resultados de la investigación expresan que la percepción del investigador frente a la realidad social, es inherente a la connotación ontológica del ser expresada entre el "idealismo y el realismo", asumiendo que el idealismo es una corriente filosófica con diversos matices que se traduce en la posición del ser como resultado de su pensar para percibir la realidad tomando un carácter subjetivo, mientras que el realismo es otra corriente filosófica con diversos matices que condiciona el pensar previo a la posición del ser para percibir la realidad objetivamente. En síntesis, el investigador en su abordaje pudiera adoptar dos perspectivas incompatibles de la realidad: (Idealismo/Subjetivista Vs Realismo/Objetivista).

Fuente: Matriz de Sistematización para la Triangulación Teórica, Autores (2021) 
Cuadro 3. ¿Cuál es la relación entre el investigador y lo investigado?

\begin{tabular}{|c|c|c|}
\hline POSICIÓN INVESTIGATIVA & POSICIÓN TEÓRICA & POSICIÓN FILOSÓFICA \\
\hline $\begin{array}{l}\text { Fuente 1: Si los sujetos cognoscentes sólo } \\
\text { contamos con nuestra razón y nuestros sentidos } \\
\text { para relacionarnos con los objetos por conocer en } \\
\text { todas y cada una de las posibilidades de realización } \\
\text { del espíritu humano (ciencia, arte, religión y } \\
\text { filosofía), sólo tenemos, en un primer momento } \\
\text { matemáticamente hablando dos posibilidades para } \\
\text { establecer contacto con la cuestión sobre la relación } \\
\text { de conocimiento entre el sujeto cognoscente y el } \\
\text { objeto por conocer: la razón y los sentidos. (Nava, } \\
\text { 2017). } \\
\text { Fuente 2: El cruce de las distinciones } \\
\text { "gnoseológicas" y "ontológicas" conduce a cuatro } \\
\text { enfoques epistemológicos, como campos } \\
\text { epistémicos desde donde se pueda orientar la } \\
\text { investigación científica. Se observa dentro de estos } \\
\text { campos epistémicos, los denominados enfoques } \\
\text { epistemológicos que dan cuerpo al plan de trabajo } \\
\text { tanto para la apropiación del conocimiento o } \\
\text { dominio de la teoría, como para la apropiación de la } \\
\text { información o dominio de la práctica (De Berrios \& } \\
\text { Briseño; 2008). }\end{array}$ & 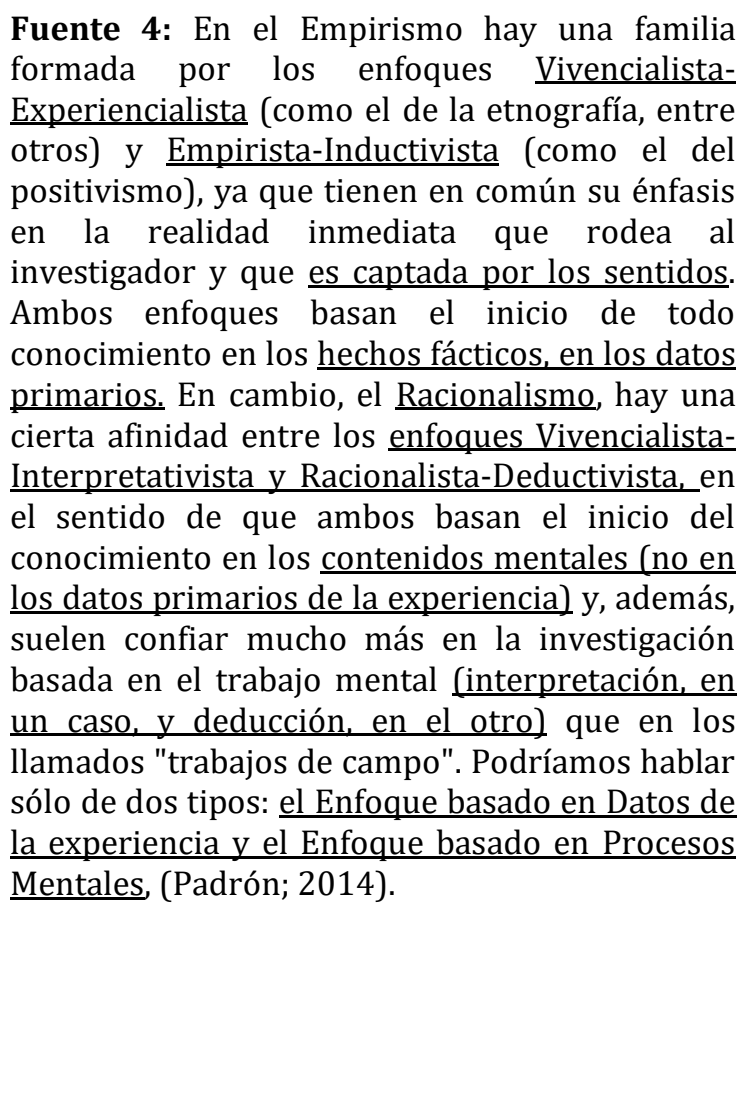 & $\begin{array}{l}\text { Fuente 6: ¿Cómo se establece la relación de } \\
\text { conocimiento entre el sujeto cognoscente y el objeto } \\
\text { por conocer? Muchos filósofos se han formulado esta } \\
\text { pregunta y la han resuelto en diferentes sentidos: para } \\
\text { algunos pensadores el sujeto cognoscente determina al } \\
\text { objeto por conocer (Descartes, 1981; Leibniz, 1991); } \\
\text { otros dicen que es el sujeto el que determina al objeto } \\
\text { (Locke, 1994, Hume, 1992) y; los más, opinan que } \\
\text { ambos elementos del conocimiento se determinan } \\
\underline{\text { recíprocamente con la salvedad de que primero se da }} \\
\text { el indeterminismo (Aristóteles, 1992; Santo Tomás de } \\
\text { Aquino, 1991) o que primero aparece el } \underline{\text { determinismo }} \\
\text { (Kant, 1996), (Nava;2017). } \\
\text { Fuente 7: El racionalismo y el empirismo desde el } \\
\text { punto de vista del problema lógico y vemos en ellos } \\
\text { dos soluciones a la cuestión de la validez del } \\
\text { conocimiento humano, llegamos a un resultado } \\
\text { semejante. Tampoco ahora podremos dar la razón al } \\
\underline{\text { racionalismo ni al empirismo. Debemos hacer, por el }} \\
\text { contrario, una distinción entre el conocimiento propio } \\
\text { de las ciencias ideales y el propio de las ciencias reales. } \\
\text { Ya la historia de ambas posiciones nos conduce a esta } \\
\underline{\text { distinción. En efecto, que los racionalistas procedían }} \\
\underline{\text { las más veces de la matemática, una ciencia ideal; los }} \\
\underline{\text { empiristas, por el contrario, de las ciencias naturales, }} \\
\underline{\text { ciencias reales. }}\end{array}$ \\
\hline
\end{tabular}




\section{POSICIÓN INVESTIGATIVA}

Fuente 3: Se describen y comentan elementos que caracterizan a los Estilos de Pensamiento de las personas (Inductivo Concreto, Deductivo Abstracto, Intuitivo Vivencial, Intuitivo no Vivencial) y como éstos se relacionan con los Enfoques Epistemológicos que muestran los científicos. En ellos, al menos uno de los tres primeros Estilos de Pensamiento se vuelve predominante, dando lugar de manera correlativa a tres Enfoques Epistemológicos: el Empirista Inductivo, el Racionalista Deductivo, Introspectivo Vivencial racionalista-subjetivista, (Yánez; 2018).

Nueva Perspectiva: Los resultados de la investigación expresan que la relación entre el investigador y lo investigado para acceder al conocimiento científico, es inherente a la postura epistemológica adoptada en el proceso investigativo, ya que se manifiestan diversas formas de abordaje para conocer la realidad en estudio, tomando en cuenta que la vía para acceder al conocimiento científico se expresa a través de procesos mentales (Racionalismo) o a través de la experiencia sensible (Empirismo). En consecuencia, se origina una relación entre la posición del investigador y la fuente del saber (Ser/Saber; Ontología/gnoseología), originando una relación Investigador/Investigado en cuatro forma predominantes: (Empirista/Inductivo, Empírico/Analítico o Empírico/Realista, atendiendo al Positivismo/Objetivismo); (Racionalista/Deductivo, Racionalista/Crítico o Racionalista/Realista, atendiendo al Postpositivimo/Objetivismo); (Introspectivo/Vivencial, Vivencialista/Experiencialista o Empírico/Idealista atendiendo al Interpretativismo/Construccionismo) y (Racional/Subjetivista, Racional/Dialectico o Racional/Idealista atendiendo al Criticismo/Subjetivista).

Fuente: Matriz de Sistematización para la Triangulación Teórica, elaborada por los autores. (2021). 
Cuadro 4. ¿Cómo se define la metodología del investigador?

\section{POSICIÓN INVESTIGATIVA}

Fuente 1: Cualquier investigación o cuantitativa o cualitativa requiere de rigor científico y ético. La rigurosidad no tiene nada que ver con la rigidez, puesto que el rigor se relaciona con el respeto por los elementos básicos de la investigación, donde se debe tener en cuenta el rigor desde lo epistémico, lo metodológico y lo teórico. El investigador debe ser consciente de su forma de ver el mundo y sus limitaciones, cumplir con las condiciones que exige el método y de la rigurosidad teórica de la cual surgen las preguntas esenciales para enunciar, (Ramírez \& Zwerg; 2012).

Fuente 2: Asimismo, es necesario considerar la relación contingente entre las maneras de recopilar la información y las preguntas de investigación (Rodríguez, Gil, García, 2006). Esto genera un sentido coherente y lógico de la metódica asumida en el proceso investigativo, y debe complementarse congruentemente con los demás aspectos del diseño propuesto. También, hay que subrayar el sentido epistemológico y ontológico de las técnicas e instrumento de recolección de información, de manera que se facilite la comunicación socializada en virtud de los hechos que se investigan, (Meléndez \& Pérez; 2006).

\section{POSICIÓN TEÓRICA}

POSICIÓN FILOSÓFICA

Fuente 4: La metodología discute los fundamentos epistemológicos del conocimiento, el papel de los valores, la idea de causalidad, el papel de la teoría y su vinculación con lo empírico, la definición y validez o aceptabilidad del recorte de la realidad, el uso y el papel que juegan la deducción e inducción, cuestiones de verificación y falsación, y los contenidos y alcances de la explicación e interpretación. No menos importante, también trata cuestiones como el papel del investigador, en general sus orientaciones culturales y especialmente sus valores, su interacción con los agentes sociales, y las diferencias y superposiciones entre los niveles macro y microsociales, (Sautu; 2005).

Fuente 5: Existe un acuerdo generalizado sobre la existencia de dos grandes marcos de referencia globales que han orientado la investigación social desde su nacimiento: la perspectiva empirista y la humanista, (las etiquetas sin muy variadas destacando entre ellas el objetivismo y subjetivismo, aquí utilizaremos el término canónico de positivismo y aunque este menos consolidado el de interpretativismo).
Fuente 6: A pesar de lo extenso del debate y las diversas acepciones en torno a la dicotimización "para acercarse a la realidad social”, es posible centrarse en la distinción más generalizada en la sociología y que se extiende a otras ciencias sociales y de la educación. La noción 'durkheniana' de investigación cuantitativa fundada en la noción objetivista de los hechos sociales a partir de lógicas explicativas, y la weberiana, a través de la cual se otorga importancia a la interpretación y comprensión de la acción social, proporcionando un papel protagónico a la subjetividad, (Cárcamo, Méndez \& Rebolledo; 2008).

Fuente 7: Lo que se pide aquí no es solo una descripción de la metodología sino también una explicación del fundamento que proporciona para la elección de métodos y las formas particulares en las que se emplean los métodos. Llevar investigación etnográfica, por ejemplo. Investigación etnográfica en el espíritu de El interaccionismo simbólico busca descubrir significados y percepciones sobre la parte de las personas que participan en la investigación, viendo stos entendimientos en el contexto de la cosmovisión general de la gente o 'cultura'. De acuerdo con este enfoque, el investigador se esfuerza por ver cosas desde la perspectiva de los participantes. 


\section{POSICIÓN INVESTIGATIVA}

Fuente 3: Defendemos la idea de que todos los cuatros dimensiones deben ser vistas como escogencia relativamente independiente en el proceso de investigación y que la escogencia cuantitativa o cualitativa es primariamente una decisión sobre la generación de datos y los métodos de análisis, solo secundariamente una escogencia sobre el delineamiento de la investigación o de intereses del conocimiento. (Bauer \& Gaskell, 2008).

Nueva Perspectiva: Los resultados de la investigación expresan que se define la metodología del investigador orientado por la realidad social que se pretende abordar y la fuente del conocimiento al que se pretende acceder, es decir; corresponde a una orientación (empirista o racionalista) en el abordaje como fuente del conocimiento, (subjetivista u objetivista) en la percepción de la realidad social, (cuantitativo o cualitativo) en la recolección de los datos de la información y (explicativa o interpretativa) en el procedimiento del investigador para valorar los resultados. En síntesis, el enfoque metodológico de la investigación "cuantitativo o cualitativo", representa la consecuencia de todo un proceso coherente que enuncia el rigor del investigador en la percepción ontológica, relación epistemológica y definición metodológica circunscrita en cada paradigma investigativo.

Fuente: Matriz de Sistematización para la Triangulación teórica, elaborada por los autores. (2021).
Se trata de dos divisiones orgánicas y claramente opuestas a la realidad social y los modos de conocerla, que han generado dos y muy diferenciada entre sí. Antes de describirlas, debemos conocer sus orígenes filosóficos para poder comprenderlas correctamente, (Corbetta; 2007).
Es esto lo que hace sentido de la intención declarada el investigador de realizar entrevistas no estructuradas y de utilizar una forma no directiva de cuestionamiento dentro de ellas, (Crotty 1998). 


\section{HERMENEUSIS DE LA INVESTIGACIÓN}

Los hallazgos de la investigación exponen que el abordaje en la investigación educativa para la aproximación, constructo, modelo o perspectiva teórica, no obedecen a una interpretación metodológica-procedimental orientada por los enfoques "cuantitativos o cualitativos"; sino que requieren una carga epistemológica que fundamenta la legitimidad en la producción científica. Según (Guba y Lincoln, 1998; p.6). Exponen que "ahora parece que ha quedado establecido más allá de cualquier objeción que las teorías y los hechos son bastante interdependientes, es decir, que los hechos sólo lo son dentro de algún marco teórico". En atención a lo expuesto, el abordaje a través de una expresión discursiva lógica consagra el modo de proceder por parte del investigador, reflejando un conjunto de enunciados donde se evidencia la percepción del investigador, la relación entre el investigador y lo investigado; al igual que la definición metodológica a utilizar en el estudio.

A nivel ontológico, el abordaje en la investigación educativa para la aproximación, constructo, modelo o perspectiva teórica debe expresar la percepción del investigador (el ser) frente al fenómeno $\mathrm{u}$ objeto de estudio, asumiendo una concepción "subjetivista $\mathrm{u}$ objetivista" para conocer la naturaleza del fenómeno a investigar, (Guba y Lincoln, 1998; p.8). Exponen "la pregunta ontológica. ¿Cuál es la forma y la naturaleza de la realidad y, por lo tanto, qué es lo que podemos conocer de ella?". En este sentido, los hallazgos del artículo de investigación reflejan que la forma $\mathrm{y}$ naturaleza en que se puede conocer la realidad es a través del (Idealismo/Subjetivista) o desde la perspectiva del (Realismo/Objetivista).
A nivel epistemológico, el abordaje en la investigación educativa para la aproximación, constructo, modelo o perspectiva teórica debe expresar la relación entre el investigador y lo investigado (el ser y el saber), tomando en cuenta la dimensión ontológica (Idealismo/Subjetivista) y (Realismo/Objetivista) con las fuentes del saber para acceder al conocimiento a través del (Racionalismo) o a través del (Empirismo), (Guba y Lincoln, 1998; p.8). Exponen "La pregunta epistemológica. ¿Cuál es la naturaleza de la relación entre quien conoce o busca conocer y lo que puede ser conocido?". En este sentido, la relación se expresa a través del abordaje epistemológicos en la investigación educativa: (Empirista/Inductivo, Empírico/Analítico o Empírico/Realista); (Racionalista/Deductivo, Racionalista/Crítico o

(Introspectivo/Vivencial,

Vivencialista/Experiencialista

0 Empírico/Idealista) y (Racional/Subjetivista, Racional/Dialectico o Racional/Idealista).

\section{MODELO TEÓRICO EN LA INVESTIGACIÓN EDUCATIVA}

El modelo teórico en la investigación educativa, se desarrolla a través del abordaje epistemológico

(Empirista/Inductivo, Empírico/Analítico o Empírico Realista), circunscrito en el "paradigma positivista". En el abordaje de la investigación educativa, se define el término "Modelo Teórico", ya que concibe el conocimiento científico como una exacta reproducción inmutable de la realidad que puede ser replicada en cualquier tiempo y espacio. En este sentido, el investigador asume una posición objetiva de la realidad donde la aprehensión del conocimiento se expresa fuera de los valores y prejuicios que 
posea el investigador, siendo validados por la experiencia sensible al comprobar la hipótesis a través de la verificación empírica en una relación sujeto-objeto.

La connotación ontológica (Ser), adopta una posición "realista" mejor conocida como "realismo ingenuo", que se expresa en la captación fiel y exacta de los hechos materiales comprobables donde destacan epistemólogos y filósofos actuales de la talla de Russell, Moore y Bunge, esta corriente filosófica se combina con la connotación Gnoseológica (Fuente del Saber), sustentando el conocimiento a través del "empirismo" que sostiene que todo conocimiento se basa en la experiencia donde destacan los filósofos Locke, Hume y J.S. Mill. En este sentido, la combinación de estas corrientes filosóficas acentuó las bases epistemológicas para el positivismo, una corriente filosófica que data desde el siglo XIX y promueve adaptar los métodos de las ciencias naturales a las ciencias sociales.

Augusto Comte (1798-1857), fue una de los filósofos más destacados de esta corriente al promulgar su obra "Curso de Filosofía Positiva” (1830-1842), para Comte se destacan tres estados en la evolución del espíritu humano, donde se expresan el estado teológico, el estado metafísico y el estado positivo. Las posturas de Comte afirmaron que el último estado promulgado o estado positivo, llevaría al descubrimiento de leyes naturales y al dominio de la naturaleza a través del hombre.

Posteriormente en 1920 surge el circulo de Viena, una corriente filosófica denominada positivismo lógico que se alejó de las posturas metafísicas incorporando premisas cientificistas donde el significado de proposición es un modo de verificación a través de la experiencia, en esta corriente destacan epistemólogos y filósofos de la talla de Schlick, Carnap, Neurath, Frank, Kauffman, Gödel, entre otros. Actualmente, el "paradigma positivista", se ha convertido en el dominante de la investigación educativa.

\section{APROXIMACIÓN TEÓRICA EN LA INVESTIGACIÓN EDUCATIVA}

La aproximación teórica en la investigación educativa, se desarrolla a través del abordaje epistemológico (Racionalista/Deductivo, Racionalista/Crítico o Racionalista/Realista), circunscrito en el "paradigma postpositivista". En el abordaje de la investigación educativa, se define el término "Aproximación Teórica", ya que concibe el conocimiento desde conjeturas o hipótesis que son aceptadas temporal o provisionalmente mientras resisten las pruebas de contrastación o falsación teórica, es decir; una teoría o hipótesis jamás podrá ser verificada ya que no existen verdades absolutas, pues siempre será posible su futura refutación con base en más datos, observaciones y experimentos. En este sentido, el investigador concibe una realidad relativa donde cualquier proposición debe someterse a revisión, es por ello; que todas las teorías se convierten en una aproximación.

La connotación ontológica (Ser), adopta una posición "realista" mejor conocida como "realismo crítico", que se expresa en someter a pruebas las teorías existentes a través del ensayo y error, conjetura o refutación para falsearlas y proponer nuevas aproximaciones teóricas más cercanas a la realidad. Su máximo exponente es el filósofo Karl Popper (1902-1994), quién reemplaza la verificabilidad positivista por la falsabilidad postpositivista, esta corriente filosófica se combina con la connotación Gnoseológica 
(Fuente del Saber), sustentando el conocimiento a través del "racionalismo", donde el saber se obtiene a través de la razón mediante la deducción objetiva, además de eso Popper destaca el papel de la experiencia empírica en el marco de la refutación teórica $y$ se le asigna el nombre de racionalismo crítico como una corriente filosófica moderna del racionalismo.

El enfoque epistemológico de Karl Popper, se expone en su obra la lógica de la investigación científica (1935), Conjeturas y Refutaciones (1963) y El conocimiento Objetivo (1972), sin embargo, en el Tercer congreso internacional de Lógica, Metodología y Filosofía de la Ciencia (1967), Popper expone "La Epistemología sin un sujeto que lo sepa", donde destaca una doctrina que manifiesta la interacción de los tres mundos del conocimiento. En el mundo 1, se conciben los objetos (el mundo universal), en el mundo 2, se conciben el mundo de los procesos mentales consientes e inconscientes (el subjetivo) y en el mundo 3 , se concibe el conocimiento objetivo (los productos de la mente humana). Así, Popper plantea la interacción de los tres mundos para percibir la realidad, valorar la experiencia y razonar con la deducción; sosteniendo el rigor del "paradigma postpositivista".

\section{CONSTRUCTO TEÓRICO EN LA INVESTIGACIÓN EDUCATIVA}

El constructo teórico en la investigación educativa, se desarrolla a través del abordaje epistemológico (Introspectivo/Vivencial, Vivencialista/Experiencialista $\quad$ o Empírico/Idealista), circunscrito en el "paradigma interpretativo". En el abordaje de la investigación educativa, se define el término de "Constructo Teórico", ya que se concibe el conocimiento a través de una construcción social que permite interpretar la realidad a través de la interacción entre los seres humanos, valorando las experiencias significativas de los contextos particulares mediados por las condiciones de tiempo y de espacio. En este sentido, el investigador concibe una realidad múltiple donde convergen diversos factores que permiten interpretar la realidad apreciando al ser en el mundo de un construccionismo compartido; recogiendo una construcción colectiva del significado y las experiencias vividas.

La connotación ontológica (Ser), adopta una posición "idealista" mejor conocida como "idealismo trascendental", quién concibe que el conocimiento humano está compuesto por juicios sintéticos apriori, es decir; sobre la base de una construcción intersubjetiva entre la sensibilidad y el entendimiento que requiere una interacción cognoscitiva para formar el juicio de valor en la mente del sujeto. Su máximo exponente es el filósofo Immanuel Kant (1724-1804), quién expone su máxima obra "Critica de la Razón Pura" (1781). Esta corriente al combinarse con la Gnoseología (Fuente del Saber), se soporta en el "Empirismo", para obtener el conocimiento a través de la experiencia sensible.

En oposición a las posturas filosóficas de Kant, surge la figura de Hegel (1770-1831), inclinando su posición filosófica hacia el "idealismo objetivo", asumiendo que la realidad puede ser percibida desde una conciencia individual destacando el principio de la razón en una determinada sociedad. Posteriormente la doctrina fenomenológica trasciende a través de Husserl (1859-1938), quién combate los argumentos de la fenomenología del espíritu (1807) de Hegel, siendo crítico del psicologismo proponiendo la "fenomenología Trascendental" (1935), tomando en cuenta la concepción del ser del 
mundo desde una referencia Cartesiana "Ideas para una fenomenología Pura" (1913) y "Meditaciones Cartesianas" (1931).

En consecuencia, Heidegger (1889-1976), discípulo de Husserl se distancia de su maestro y orienta su filosofía hacia la interpretación del ser en la realidad social y humana mediado por su temporalidad "ser en el mundo", con la promulgación de su obra Ser y Tiempo (1927), fundamentando las bases del "paradigma fenomenológicohermenéutico".

\section{PERSPECTIVA TEÓRICA}

La perspectiva teórica en la investigación educativa se desarrolla a través del abordaje epistemológico (Racional/Subjetivista, Racional/Dialéctico o Racional/Idealista), circunscrito en el paradigma crítico. En el abordaje de la investigación educativa, se define el término "Perspectiva Teórica", ya que el investigador está vinculado con su fenómeno en estudio inextricablemente y fija su posición investigativa desde una crítica social emancipadora mediada intersubjetivamente por los símbolos significantes del lenguaje en la acción comunicativa, para concebir una realidad que puede ser cuestionada en el mundo de la vida y los sistemas sociales. En consecuencia, el investigador procura la emancipación a través de la reflexión crítica del saber y la racionalidad expresada en la acción comunicativa.

La connotación ontológica (Ser), adopta una posición "idealista" mejor conocida como "idealismo objetivo", donde el conocimiento se concibe como un concepto único fundamentado en la razón, ya que está vinculado a un orden histórico definido con un trasfondo político. Su máximo exponente es Georg Hegel (1770-1831), quién fundamentó su pensamiento filosófico en las obras Fenomenología del Espíritu (1807), La Ciencia de la Lógica (1812) y la Filosofía de la Historia (1837), convirtiéndose en un crítico del idealismo trascendental propuesto por Kant (1724-1804). Hegel concibe la naturaleza humana en términos históricos impulsada por el comportamiento instintivo hacia el comportamiento racional a través de un proceso histórico denominado proceso dialéctico. Esta corriente al combinarse con la Gnoseología (Fuente del Saber), expresa un fundamento del saber desde el "racionalismo" donde la vía del conocimiento prevalece en la intuición del pensamiento.

Desde la basa Hegeliana se desarrollaron un conjunto heterogéneo de corrientes filosóficas que imprimieron diversos matices entre las cuales destacan: El Estructuralismo, Posestructuralismo y Postmodernismo, destacando la relevancia de escuela de Frankfurt (Alemania), con filósofos de la talla Max Horkheimer, Adorno, Marcuse y Fromm

En la segunda generación de la escuela de Frankfurt, surgen filósofos de la talla de Jürgens Habermas (1922). La cual, expone la actualización de la teoría crítica en su obra la acción comunicativa (1981), superando un cúmulo de contradicciones de esta corriente filosófica en el pasado entre los materialistas y los trascendentales, en esta perspectiva Habermas asume el idealismo objetivista mediado por la acción comunicativa del lenguaje en una relación intersubjetiva del saber, asignando una actualización del "paradigma crítico".

\section{CONSIDERACIONES FINALES}

Finalmente, la investigación considera que el abordaje para la aproximación, constructo, modelo o perspectiva teórica, debe resguardar una coherencia interna 
discursiva, perceptiva, relacional $\mathrm{y}$ procedimental, tomando en cuenta el plano ontológico, epistemológico y metodológico de la investigación para legitimar el conocimiento científico.

La percepción del investigador frente a la realidad social, es inherente a la connotación ontológica del ser expresada entre el "idealismo y el realismo", asumiendo que el idealismo es una corriente filosófica con diversos matices que se traduce en la posición del ser como resultado de su pensar para percibir la realidad tomando en cuenta un carácter subjetivo del investigador, mientras que el realismo es otra corriente filosófica con diversos matices que condiciona el pensar previo a la posición del ser para percibir la realidad objetivamente. En tal sentido, el investigador en su abordaje pudiera adoptar dos perspectivas incompatibles de la realidad: (Idealismo/Subjetivista

Vs

Realismo/Objetivista).

La relación entre el investigador y lo investigado para acceder al conocimiento científico, es inherente a la postura epistemológica adoptada en el proceso investigativo, ya que se manifiestan diversas formas de abordaje para conocer la realidad en estudio. En consecuencia, se origina una relación entre la posición del investigador y la fuente del saber (Ser/Saber; Ontología/gnoseología), originando una relación Investigador/Investigado en cuatro formas predominantes: (Empirista/Inductivo, Empírico/Analítico o Empírico/Realista); (Racionalista/Deductivo, Racionalista/Crítico o Racionalista/Realista);

(Introspectivo/Vivencial,

Vivencialista/Experiencialista

0

Empírico/Idealista) y (Racional/Subjetivista, Racional/Dialectico o Racional/Idealista).
La metodología del investigador está orientada por la realidad social que se pretende abordar y la fuente del conocimiento al que se pretende acceder, es decir; corresponde a una orientación (empirista o racionalista) en el abordaje como fuente del conocimiento, (subjetivista $u$ objetivista) en la percepción de la realidad social, (cuantitativo o cualitativo) en la recolección de los datos de la información y (explicativa o interpretativa) en el procedimiento del investigador para valorar los resultados.

\section{REFERENCIAS}

Bauer, M. \& Gaskell, G. (2008). Pesquisa Qualitativa com Texto: Imagem e Som: Um Manual Prático; Tradução de Pedrinho A. Guareschi. 7ma Edição. Rio de Janeiro: Ed. Petrópolis. Recuperado de https://tecnologiamidiaeinteracao.files.w ordpress.com/2017/10/pesquisaqualitativa-com-texto-imagem-e-sombauer-gaskell.pdf (Consultado en Enero, 2021)

Camacho, H; Fontaines, T. \& Urdaneta, G. (2005). La Trama de la Investigación y su Epistemología. (Revista en Línea). Vol. 7. Núm. 1. Recuperado de https://www.redalyc.org/pdf/993/99318 830001.pdf (Consultado en Enero, 2021)

Cárcamo, H; Méndez, P. \& Rebolledo, A. (2008). Expresión del Debate Cuantitativo/Cualitativo en las Ciencias de la Educación. I Encuentro Latinoamericano de Metodología de las Ciencias Sociales, 10, 11 y 12 de diciembre de 2008, La Plata, Argentina. En Memoria Académica. Recuperado de http://www.memoria.fahce.unlp.edu.ar/t rab_eventos/ev.9463/ev.9463.pdf (Consultado en Enero, 2021)

Carnap, R. (1957). Conocimiento y verdad. En: El Positivismo Lógico. Fondo de Cultura Económica, México 
Chaves, A; Gadea, W. (2018). La Relación Subjeto-Objeto en la Concepción Kantiana de la Ciencia. (Revista en Línea). Vol. 1. Núm. 25. Recuperado de https://www.redalyc.org/jatsRepo/4418 /441855948003/html/index.html

(Consultado en Enero, 2021)

Comte, A. (1981) [1830], Curso de Filosofía Positiva. Primera y Segunda lecciones. Buenos Aires: Aguilar

Corbetta, P. (2007). Metodología y Técnicas de Investigación Social. España: McGraw Hill. Recuperado de https://luisdoubrontgschool.files.wordpr ess.com/2021/04/corbetta-metodologiay-tecnicas-de-investigacion-social.pdf (Consultado en Enero, 2021)

Crotty, M. (1998). The Foundations of Social Research. London: SAGE Publications. Recuperado de https://seminariodemetodologiadelainve stigacion.files.wordpress. com/2012/03/the-foundations-of-socialresearch-meaning-and-perspective-inthe-research-process-michael-crotty.pdf (Consultado en Enero, 2021)

De Berrios, O. \& Briceño, M. (2009). Enfoques Epistemológicos que Orientan la Investigación de 4to. Nivel. (Revista en Línea). Vol. 1. Núm. 8. Recuperado de https://www.redalyc.org/pdf/4655/4655 45882009.pdf (Consultado en Enero, 2021)

Foucault, M. (1970). La Arqueología del Saber. Paris: Editions Gallimard. Recuperado de https://luisdoubrontgschool.files.wordpr ess.com/2021/04/foucault-michel-laarqueologia-del-saber.pdf (Consultado en Enero, 2021)

Galeano, M. (2012). Estrategias de Investigación Social Cualitativa. Medellín: La Carreta Editores. Recuperado de https://luisdoubrontgschool.files.wordpr ess.com/2021/04/galeano-m.-2004.estrategias-de-investigacion-socialcualitativa.pdf (Consultado en Enero, 2021)

Guba, E. \& Lincoln, Y. (1998). Competing Paradigms Qualitative Research, en
Denzin, N.K. \& Y.S. Lincoln, The Landscape Qualitative Research, Thousand Oaks, CA: Sage. Recuperado de http://www.hugoperezidiart.com.ar/siglo XXI-cl2012/guba-lincoln-1998.pdf (Consultado en Enero, 2021)

Habermas, J. (1999); [1981]. Teoría de la acción comunicativa. Traducido por Manuel Jiménez Redondo. Madrid: Taurus

Hegel, G.W.F. (1807). System der Wissenschaft. Erster Teil, die Phänomenologie des Geistes, Bamberg und Würzburg

Hegel, G.W.F. (2011); [1812]. Ciencia de la Lógica. Traducido por Félix Duque. Madrid: Abada Editores

Hegel, G.W.F. (2016); [1837]. Filosofía de la Historia. Madrid: Ediciones Losada

Heidegger, M. (1996); [1927]. Ser y Tiempo. Traducción de Jorge Eduardo Rivera. Madrid: Trotta

Hessen, J. (1999). Teoría del Conocimiento. San José de Costa Rica: ILCA. Recuperado de

https://trabajosocialucen.files.wordpress. com/2012/05/hessen_johannes_teoria_de l_conocimiento_pdf-1.pdf (Consultado en Enero, 2021)

Hincapié, J. (2017). Elementos Ontológicos, Epistemológicos y Metodológicos para la Construcción de un Marco Teórico de Estudio de los Intangibles. (Revista en Línea). Vol. 18. Núm. 45. Recuperado de http://www.scielo.org.co/pdf/cuco/v18n 45/0123-1472-cuco-18-45-00086.pdf (Consultado en Enero, 2021)

Horkheimer, M. (2000); [1972]. Teoría Tradicional y Teoría Crítica. Traducido por José Luis López y López de Lizaga. Barcelona: Paidós

Husserl, E. (1984); [1935].Crisis de las ciencias europeas y la fenomenología trascendental, México, Ediciones Folios, (El manuscrito data de 1935-1936, publicado originalmente en alemán en 1962)

Husserl, E. (1985); [1931]. Meditaciones Cartesianas. Madrid: Fondo de Cultura Económica. 
Husserl, E. (1993); [1913]. Ideas Relativas a una Fenomenología Pura y una Filosofía Fenomenológica. Madrid: Fondo de cultura económica

Kant, I. (2000); [1781]. Crítica de la Razón Pura. Madrid: Alfaguara

Meléndez, L. \& Pérez, C. (2006). Propuesta Estructural para la Construcción Metodológica en Investigación Cualitativa como Dinámica del Conocimiento Social. (Revista en Línea). Vol. 3. Núm. 3. Recuperado de http://ve.scielo.org/scielo.php?script=sci_ arttext\&pid=S1690-75152006000300003 (Consultado en Enero, 2021)

Nava, J. (2017). La esencia del Conocimiento. El Problema de la Relación Sujeto-Objeto y Sus Implicaciones en la Teoría Educativa. (Revista en Línea). Vol. 8. Núm. 15. Recuperado de https://pdfs.semanticscholar.org/a2b5/8f aa36b4ed83687887affef92453af5f2b96.p df (Consultado en Enero, 2021)

Ortiz, E. (2013). Epistemología de la Investigación Cuantitativa y Cualitativa: Paradigmas y Objetivos. (Revista en Línea). Vol. 2. Núm. 408. Recuperado de https://www.researchgate.net/publicatio n/303313448_Epistemologia_de_la_Invest igacion_Cuantitativa_y_Cualitativa_Paradig mas_y_Objetivos (Consultado en Enero, 2021)

Padrón, J. (2014). Proyecto de Epistemología en DVD. Doctorado en Ciencias Humanas, Maracaibo: La Universidad del Zulia. Recuperado de http://padron.entretemas.com.ve/Notas_ EP-EnfEpistPdigmas.pdf (Consultado en Enero, 2021)

Paz-Sandín, E. (2003). Investigación Cualitativa en Educación. Fundamentos y Tradiciones. Madrid: McGraw Hill. Recuperado de https://luisdoubrontgschool.files.wordpr ess.com/2021/03/paz-sandinesteban_investigacion-cualitativa-eneducacion_-.pdf (Consultado en Enero, 2021)

Perdomo, E. (2009). Michael Foucault La
Arqueología del Saber. (Revista en Línea). Vol. 1. Núm. 3. Recuperado de http://www.saber.ula.ve/bitstream/hand le/123456789/28600/articulo9_3.pdf?se quence $=1$ \&isAllowed $=\mathrm{y}$ (Consultado en Enero, 2021)

Popper, K. (1935). Logik der Forschung. Vienna: Julius Springer Verlag

Popper, K. (1963). Conjectures and Refutations: The Growth of Scientific Knowledge. Routledge: London

Popper, K. (1967). Epistemología sin sujeto que sabe, en Lógica, metodología y filosofía de la ciencia III, B. Van Rootselaar y JF Staal (eds.), (Estudios de lógica $\mathrm{y}$ fundamentos de las matemáticas 52), Amsterdam: Elsevier, 333-373. Reimpreso en Popper 1972a: 106152. Doi: 10.1016 / S0049-237X (08) 71204-7

Popper, K. (1972). Objective Knowledge: An Evolutionary Approach. Oxford: Clarendon Press

Ramírez, F. \& Zwerg, A. (2012). Metodología de la Investigación: Más que una Receta. (Revista en Línea). Vol. 12. Núm. 20. Recuperado de https://core.ac.uk/download/pdf/29065 1523.pdf (Consultado en Enero, 2021)

Sautu, R. (2005). Todo es Teoría. Objetivos y Métodos de Investigación. Buenos Aires: Lumiere. Recuperado de https://luisdoubrontgschool.files.wordpr ess.com/2021/04/ruth-sautu-

odo_es_teoria_objetivos_y_metodos_en_in vestigacion_sautu_ruth.pdf (Consultado en Enero, 2021

Severini, L. (1961). Existencialismo. Barcelona: Herder

Torres, A. \& López, D. (2014). Criterios para Publicar Artículos de Revisión Sistemática. (Revista en Línea). Vol. 19. Núm. 3. Recuperado de https://www.medigraphic.com/pdfs/quir urgicas/rmq-2014/rmq143t.pdf (Consultado en Enero, 2021)

Vidal, J. (2013). La Búsqueda de la Realidad o de la Verdad: Una Aproximación a partir de la Teoría Sociológica. (Revista en 
Línea). Vol. 1. Núm. 47. Recuperado de https://scielo.conicyt.cl/scielo.php?script =sci_arttext\&pid=S0717-

554X2013000200004 (Consultado en Enero, 2021)

Yánez, P. (2018). Estilos de Pensamiento, Enfoques Epistemológicos y la Generación del Conocimiento Científico. (Revista en Línea). Vol. 39. Núm. 51. Recuperado de https://www.revistaespacios.com/a18v3 9n51/a18v39n51p18.pdf (Consultado en Enero, 2021) 